infarction (MI), target lesion revascularization (TLR) and definite or probable stent thrombosis (ST). We also compared binary angiographic restenosis (BAR) at 6-8 months.

Results The median follow up for the entire study cohort was 10.0 years [interquartile range, 9.8 to 10.0 years]. At 10 years, all-cause mortality $(\mathrm{HR}=1.05$ [0.95-1.16]; $\mathrm{p}=0.348) \mathrm{did}$ not differ between the stent overlap and no stent overlap groups. MI $(8.4 \%$ vs $5.2 \% ; \mathrm{HR}=1.67$ [1.35-2.07], $\mathrm{p}<0.001)$ and $\operatorname{TLR}(23.7 \%$ vs $16.3 \% ; \mathrm{HR}=1.54$ [1.36-1.74], $\mathrm{p}<0.001)$ occurred more frequently in the stent overlap group. For MI, landmark analysis demonstrated that this increase in risk was primarily in the first 30 days post PCI, with comparable outcomes from 30 days to 10 years. Definite/probable ST was numerically higher in the stent overlap group $(\mathrm{HR}=1.45$ [0.98-2.17], $\mathrm{p}=0.06)$. BAR at 6-8 months was also more frequent in the stent overlap group $(16.0 \%$ vs $10.3 \% ; \mathrm{HR}=1.65$ [1.41-1.92], $\mathrm{p}<0.001)$.

Conclusion DES overlap is associated with an increased risk of adverse clinical events post PCI.

\section{AUDIT OF PRESCRIBING PRACTICES IN WOMEN OF CHILD BEARING AGE IN GENERAL CARDIOLOGY CLINIC IN BEAUMONT HOSPITAL}

C Condon, N Humphreys, BF McAdam. Beaumont Hospital, Dublin, Ireland

10.1136/heartjnl-2021-ICS.53

Background In 2018, The European Society of Cardiology released a clinical practice guideline on the management of cardiovascular disease in pregnancy and made specific recommendations on need for care with drug therapies before and during pregnancy. Given that up to $25 \%$ of Irish pregnancies are unplanned, the risk benefit of prescribing any potentially teratogenic drug must be considered and discussed with the patient. We aimed to identify prescription patterns of cardiology medications to women of child bearing age and to identify if potential teratogenicity was highlighted and to identify if appropriate advice of family planning, contraception or breastfeeding was offered and documented.

Methods We reviewed clinic letters of women under the age of 50 attending cardiology clinic, as well as the Heart Support Unit (HSU) in Beaumont Hospital between January 2019 and January 2020. The information gathered includes; MRN, age, cardiovascular medications prescribed, documentation of the risk benefit of prescriptions, documentation of family planning or breast-feeding.

Results 331 patient clinic letters were reviewed as a sample of a total of 695 women under 50 who attended cardiology clinic in the period, with $2.8 \%$ attending HSU. The mean age was 37.9 years (SD 8.6). The majority (51\%) were aged between 40 and 50. 29.9\% were aged between 30 and 39 . $59.8 \%$ of women were not prescribed any medications. $23 \%$ of this cohort were prescribed one medication and beta blockers were the most frequently prescribed medication (23.6\%). Within beta blocker prescriptions, 76\% were bisoprolol, 6\% metoprolol, 5\% labetalol, 3\% sotalol, 3\% nebivolol and 1\% each for atenolol, propranolol and carvedilol. 45 (13\%) women were prescribed either an ACE inhibitor or an ARB. $27(8 \%)$ women were prescribed a statin, $23(6 \%)$ were prescribed an antiplatelet agent. Figure 1 demonstrates the frequency drugs were prescribed. Of the five women who attended the HSU and documents were reviewed, all were prescribed three or more medications and one patient was
Drug Prescription Frequency

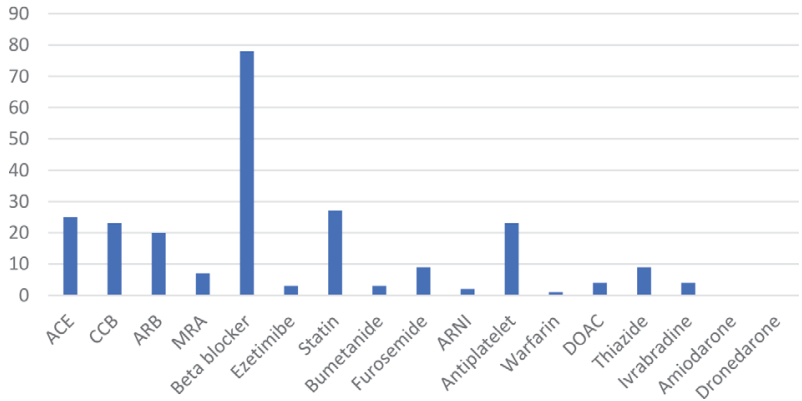

Abstract 53 Figure 1 Frequency of drug prescription

prescribed six medications. None of these prescriptions were associated with any documentation of teratogenic risk or family planning. Of the 331 patient documents, 11 mentioned family planning intentions, pregnancy or potential risks of $\mathrm{CV}$ medication use during pregnancy. Issue of breastfeeding was not documented in any case.

Conclusions In women of childbearing age attending the cardiology department, $40 \%$ are prescribed medications, however associated documentation of issue of pregnancy, contraception, family planning intentions, breastfeeding is low. Documentation of potential teratogenic risks associated with these medications is also low. Patients with complex polypharmacy attending the HSU were not appropriately advised of the compounded teratogenic risk. Clinicians require more education in this regard and this aspect of the clinical encounter in this population requires proper documentation

\section{CARDIOLOGY ADVANCED NURSE PRACTITIONER (ANP) REFERRAL FOR CORONARY ANGIOGRAM: AN INITIAL EXPERIENCE}

P Stoneman, F Colbert, J Adams, M Kennedy, H Hussein, R Sheahan, BF McAdam, T Gumbrielle, I Yearoo, E Fahy. Beaumont Hospital, Dublin, Royal College of Surgeons in Ireland, Dublin, Ireland

\subsection{6/heartjpl-2021-ICS.54}

Introduction The Emergency Cardiology Service is a front line ANP led, consultant directed, protocol driven, clinical service focused on treating often high acuity patients with a wide range of general cardiac complaints early in their emergency department journey. Our service provides expert consult, diagnosis, and treatment, facilitating early discharge to outpatient ambulatory diagnostics/ANP led Rapid-access Virtual Clinic, or admission for inpatient diagnostics. In the 17 years prior to February 2021, patients determined to have a high pre-test probability of coronary disease based upon their typicality of symptoms, risk profile, ECG and biomarkers, would be discussed with the consultant cardiologist prior to an angiogram being ordered to ensure the test was justified. In 2020, the NMBI expanded the referring scope of practice for nurses referring for ionising radiation to include all medical radiological and other imaging procedures for nurses who had completed a comprehensive post graduate level 8 award, containing both academic and clinical components as set down by NMBI (NMBI, 2020) and contained within SI 256 of 2018. In order to enhance our service, we developed additional standard operating procedures (SOPs) [figure 1], [figure 2] which led to a memorandum of understanding on 6th 
February 2021 between the consultant cardiology group and radiology department, to allow our cardiology ANP group to refer for coronary angiogram, making our ANP service the first in Ireland to refer for coronary angiogram.

Methodology A retrospective audit of referrals for coronary angiogram made by the ANP service to the consultant group from February 6th 2021 were audited to ensure adherence to our SOP and SI256 with regards to the principle of justification including: $\bullet$ Have a corresponding signature $\bullet$ Have evidence of prior thorough examination $\bullet$ Have a clear justification - Adhere to local and national protocols • Adhere to radiation safety policies.

Results 67 in-patient and out-patient coronary angiogram requests were made on McKesson radiology or referred by letter to private rooms by the ANP service between 6th February to date. All coronary angiograms with deemed justified by the practitioner (consultant cardiologist) and subsequently completed. All ANP requests adhered to our SOP and met the standards in relation to the principle of justification set out in SI256.

Conclusion Our initial experience of cardiology ANP as referrer for coronary angiogram is positive. We have demonstrated that working within our scope of practice framework and standard operating procedures has ensured that all referrals were considered justified by the consultants who ultimately performed the tests.

Implications Cardiology ANP referral for coronary angiogram has the potential to safely streamline the management of

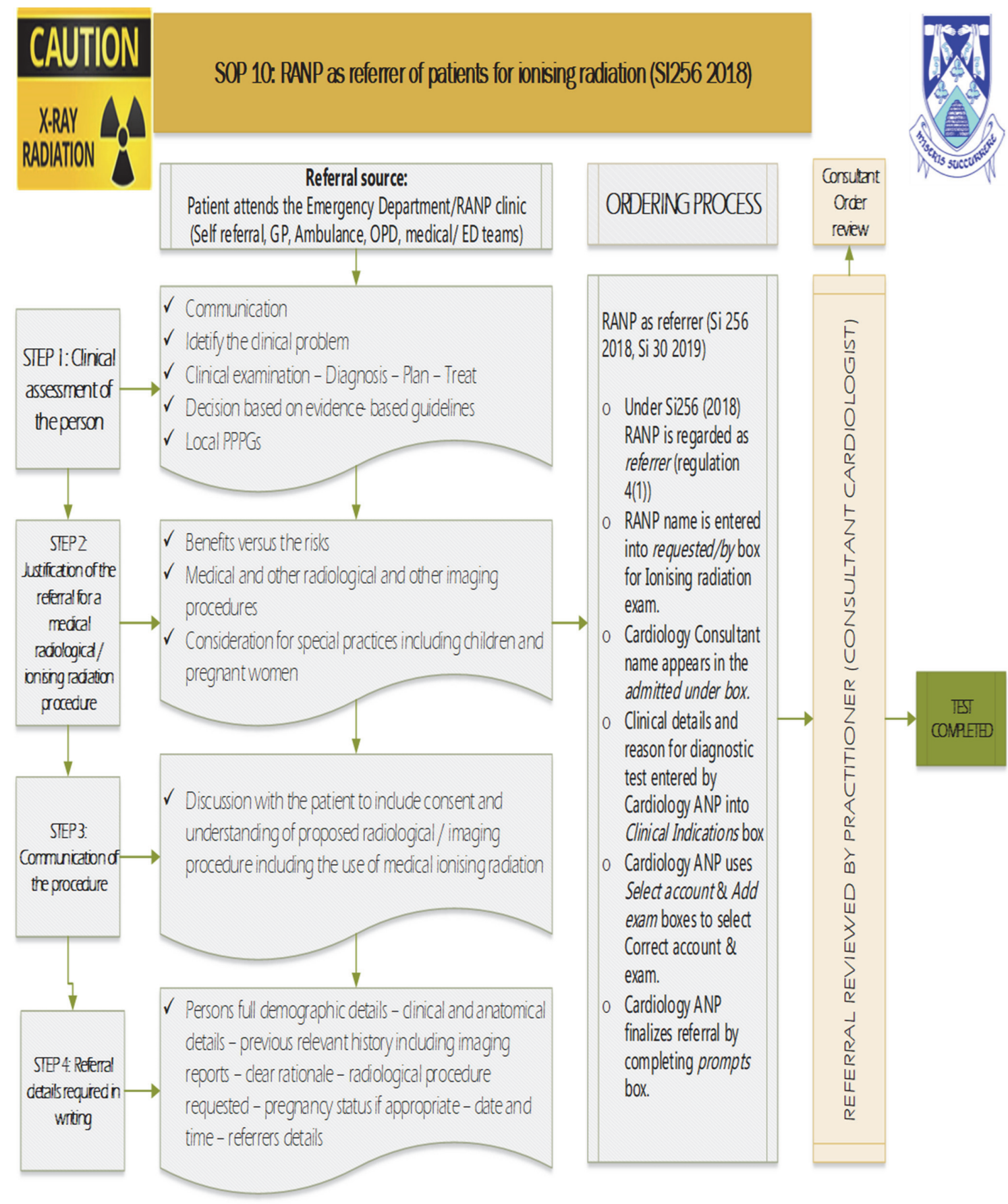




\section{SOP 9: RANP self assessment of referral for radiological procedures}

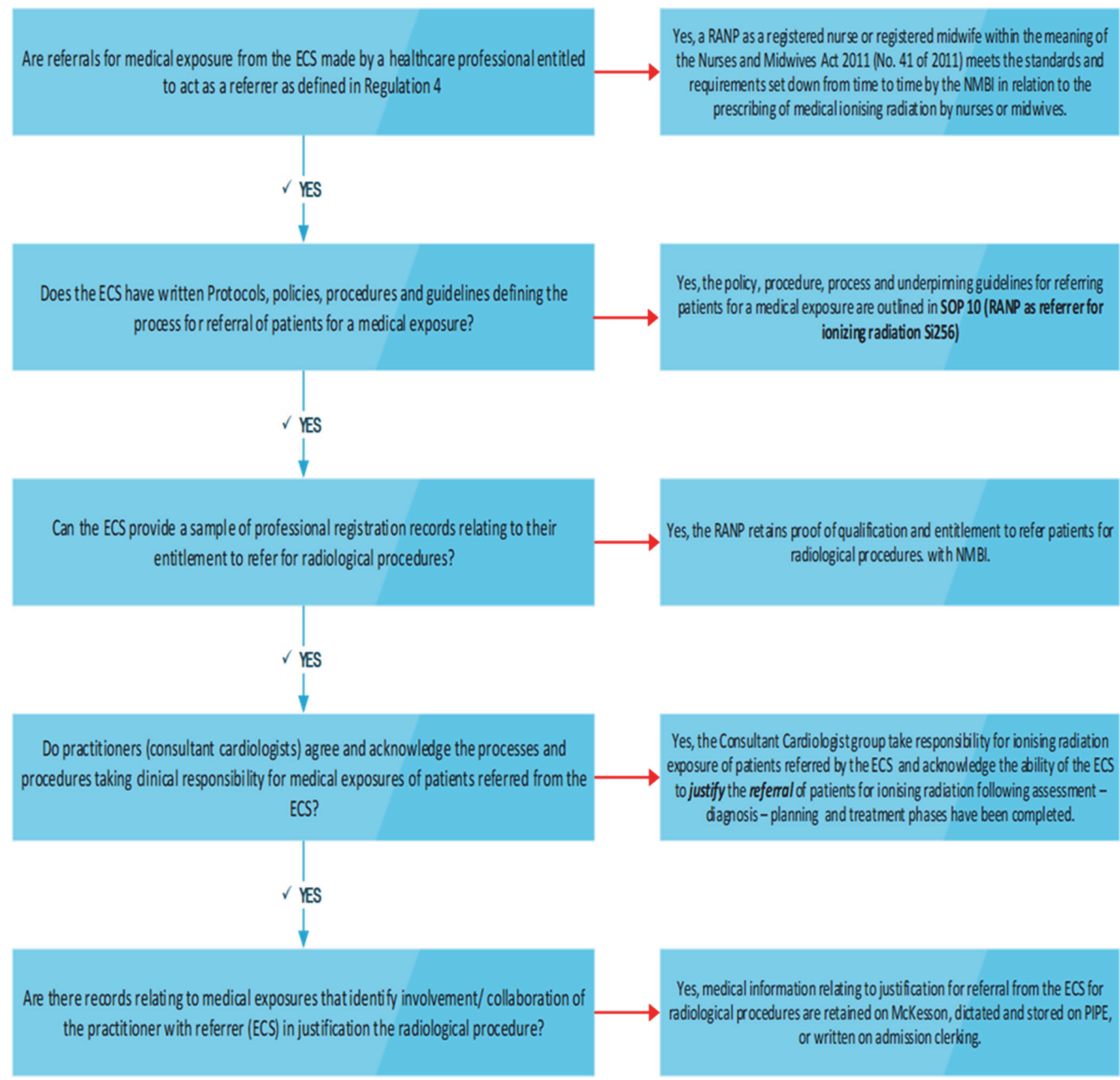

Abstract 54 Figure 2 SOP 9

patients across the country provided there is consultant support, adequate training/education and standard operating procedures are in place prior to commencement.

\section{COMPARATIVE EFFICACY AND SAFETY OF NON- VITAMIN K ORAL ANTICOAGULANTS: A QUALITATIVE REVIEW OF CLINICAL EVIDENCE}

\footnotetext{
${ }^{1,2} \mathrm{~A}$ Jordan, ${ }^{1,3} \mathrm{H}$ Rai, ${ }^{1,3} \mathrm{R}$ Colleran, ${ }^{1,4} \mathrm{RA}$ Byrne. ${ }^{1}$ Cardiovascular Research Institute Dublin, Mater Private Network, Ireland; ${ }^{2} R C S I$ University of Medicine and Health Sciences, Dublin, Ireland; ${ }^{3}$ School of Medicine, RCSI University of Medicine and Health Sciences, Dublin, Ireland; ${ }^{4}$ School of Pharmacy and Biomolecular Sciences, RCSI University of Medicine and Health Sciences, Dublin, Ireland
}

Introduction Non-vitamin $\mathrm{K}$ antagonist oral anticoagulants (NOACs) were approved for clinical use for prevention of stroke or systemic embolism in patients with atrial fibrillation, or for prevention of venous thromboembolism in hip or knee replacement surgeries based on pivotal clinical trials comparing safety and efficacy versus vitamin $\mathrm{K}$ antagonist therapy. However, large-scale randomized controlled trials directly comparing individual approved agents is a notable scientific gap. The aim of this analysis was to provide a narrative review of studies that have provided direct comparisons of safety or efficacy of different NOACs.

Methods We did a systematic review of studies investigating comparative efficacy and/or safety of NOACs approved for use in Ireland using Medline/PubMed with publication date between January 2000 and December 2020. Original studies involving quantitative comparisons of safety or efficacy 